\title{
Development of the Scheme of the Installation for Mechanical Wastewater Treatment
}

\author{
Ivan V. Sevostianov ${ }^{1}$, Yaroslav V. Ivanchuk ${ }^{1 *}$, Oleksandr V. Polishchuk ${ }^{2}$, \\ Vladyslav. L. Lutsyk ${ }^{1}$, Kateryna V. Dobrovolska ${ }^{3}$, Saule Smailova ${ }^{4}$, \\ Waldemar Wójcik ${ }^{5}$, Aliya Kalizhanova ${ }^{6}$
}

1 Department of Industrial Engineering, Vinnytsia National Technical University, 95 Khmelnytske shose str., 21021, Vinnytsia, Ukraine

2 Department of Life Safety and Security Education, Vinnytsia National Technical University, 95 Khmelnytske shose str., 21021, Vinnytsia, Ukraine

3 Department of Biophysics, Medical Equipment and Informatics, National Pirogov Memorial Medical University, 56 Pirogova Str., 21018, Vinnytsia, Ukraine

4 East Kazakhstan State Technical University named after D.Serikbayev, Naberezhnaya Krasnykh Orlov, 69, UstKamenogorsk, Kazakhstan

5 Department of Electronics and Information Technology, Lublin University of Technology, Nadbystrzycka 38a, 20-618 Lublin, Poland

6 Institute of Information and Computational Technologies CS MES RK, Almaty, Kazakhstan; University of Power Engineering and Telecommunications, Almaty, Kazakhstan

* Corresponding author's e-mail: ivanchuck@ukr.net

\begin{abstract}
The paper presents the schemes of various equipment for the mechanical treatment of the industrial and domestic wastewater, which allows removing it from the main part of the pollution. An examination of the shortcomings of the known equipment with the aim of its improvement was conducted. As a result, an installation scheme for continuous high-performance mechanical wastewater treatment was proposed. In contrast to the known equipment, the proposed installation provides a three-staged treatment of wastewater with the help of the two pairs of gratings - for preliminary treatment (removal of large-size pollutions) and by filtration through the two metallic sieves - for normal and fine treatment (removal of small-size mechanical pollutions). The installation consists of highly-efficient appliances for the cleaning of filtering elements in the course of realization of the working process, which provides stable high productivity of treatment. The installation has simple reliable design and low energy expenses. The paper contains the formulas for determining of main operational parameters of the installation: the change of the cross-sectional area of the filtering elements and productivity of the working process, periodicity of cleaning of gratings and sieves, the necessary electric power of installations drives. These formulas can be a basis for further research of installation and for elaboration of a method of its design calculation.
\end{abstract}

Keywords: industrial and domestic wastewater, continuous mechanical treatment, installation, efficiency.

\section{INTRODUCTION}

Wastewater treatment is an urgent task for countries, cities and individual enterprises, since the quality of water treatment determines the possibility of its reuse for drinking, cooking, household and production needs, in agriculture, etc. It is known that the optimal method of cleaning and the most effective equipment for its implementation are selected in each specific case, taking into account the type of sewage (industrial, domestic, atmospheric), the type of pollution (mineral, organic, mineral-organic), their concentrations (1-500, 500-5000, 5000-30 000,> 30000 mg/l), 
the physical properties of the particles of contamination, the degree of aggressiveness of the effluents and the required degree of their purification [Iskovych-Lototsky, 2018a, 2018b].

In most industrial and domestic wastewater treatment systems, one of the main stages is preliminary mechanical cleaning. This allows removing up to $60 \%$ of contaminants, including coarsely dispersed particles, at moderate energy and time costs [Iskovych-Lototsky, 2019, Jörg, 2010]. It is considered the most well-known equipment for the mechanical wastewater treatment.

Lattices and screens are the first elements of all technological wastewater treatment systems, since the largest particles can be removed from them [Iskovych-Lototsky, 2019]. In most designs, the grids are a system of parallel metal rods, between which drainage flows. At the same time, large contaminants are retained by rods, which are cleaned with mechanical rakes. Figure 1 shows a lattice scheme with wedge-shaped steel rods 1 rigidly fixed in the bottom part of the channel [Iskovych-Lototsky, 2019]. Rake 2 associated with the belt conveyor removes contamination from the rods and drops it on the conveyor belt 6 , instead of which a screw conveyor or hydraulic transport can also be used. Lattices are produced with a width of clearances of 1-50 mm and a working width of 338-1200 $\mathrm{mm}$. The main disadvantage of this equipment is the incomplete cleaning of the rods 1 and, as a result, a decrease in the productivity of the working process. In addition, such gratings are suitable only for the crudest pre-treatment of water.

Figure 2 shows a straining device - a mechanized flat slotted screen [Iskovych-Lototsky, 2019]. It consists of a frame 1 with a flat slotted grid 2 of a prefabricated structure with $1.4 \mathrm{~mm}$ clearances and a mechanism for its regeneration in the form of a belt conveyor with scrapers 3 , driven by a geared motor (not shown in the diagram). The detainees on the grid 2 are continuously removed by scrapers 3 and dumped into a container. The tests of a prototype of a sieve with a working width of $2.25 \mathrm{~m}$ showed that its productivity is three times higher than the productivity of the drum net $\left(333.000 \mathrm{~m}^{3} /\right.$ day vs. $110.000 \mathrm{~m}^{3} /$ day $)$, with significantly lower head losses $(92 \mathrm{~mm}$ vs. $300 \mathrm{~mm}$ ). As noted in [Iskovych-Lototsky, 2019], during the four-month tests of the sieve, a satisfactory regeneration of the grid and clearances in it was detected, which remained pure throughout the entire observation period. In our opinion, a disadvantage of the screen in the course of its operation is the high probability of the particles of impurities squeezing through the gaps of the scrapers. In addition, during the operation of this equipment, intense scrubbing of the scrapers takes place on the mesh, which leads to their accelerated wear and loss of power.

Another common type of wastewater treatment equipment is the sand trap. It provides

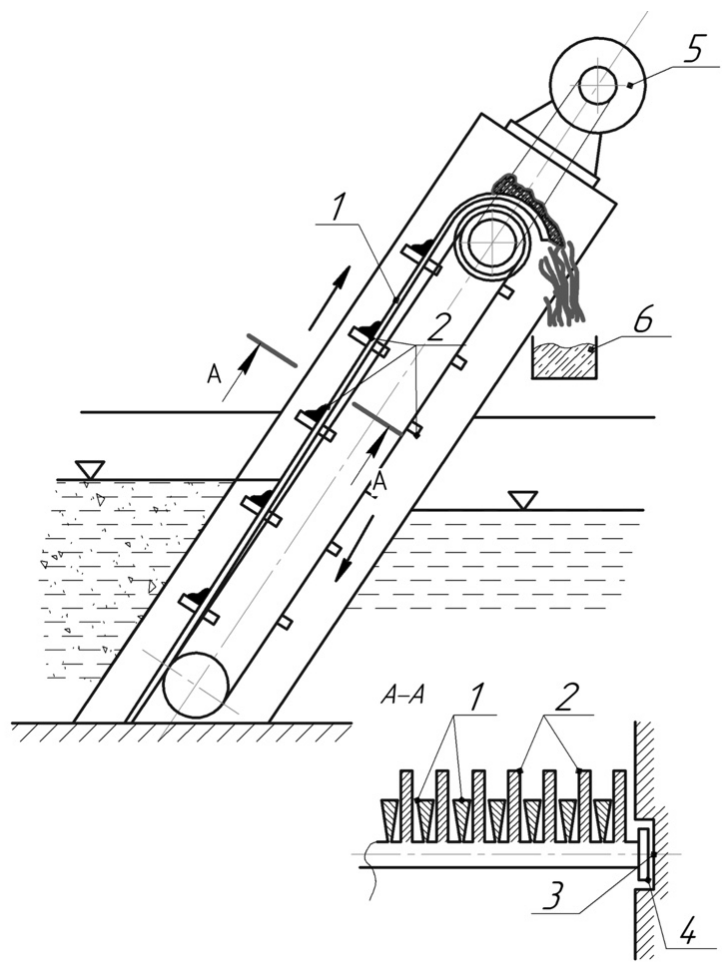

Figure 1. The lattice scheme of the firm «Jones and Attwood» (Great Britain): 1 - rods; 2 - rake; 3 - support rake; 4 - guide supports; 5 - the electric motor; 6 - belt conveyor [Iskovych-Lototsky 2019]

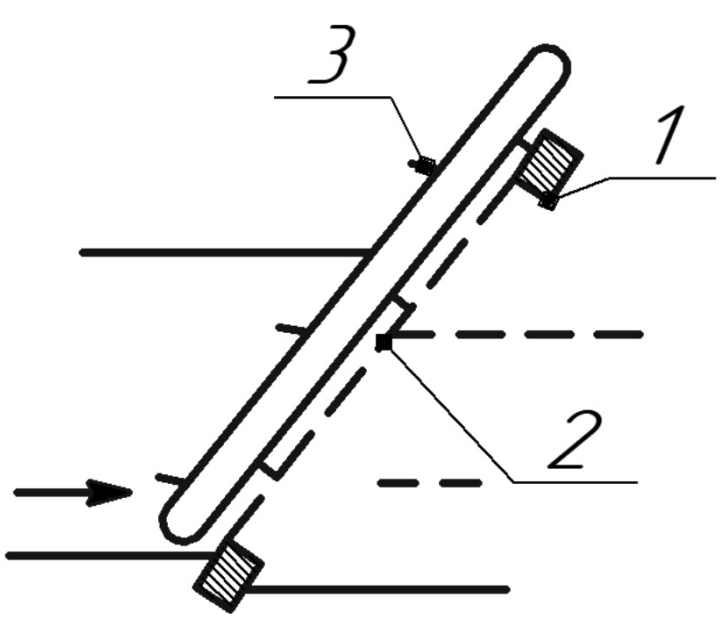

Figure 2. Scheme of a mechanized slotted screen 
separation of solid heavy mineral particles (sand, slag, glass bottles, etc.). Figure 3 shows one of the most effective horizontal sand pitches with a hydromechanical sludge removal system. The purified water passes through the flowing part of the sand trap. As a result, the solid mineral particles contained therein are deposited by gravity in the tray 2 . In order to prevent compaction of the sediment in the tray 2, which leads to an increase in the energy consumption for its transportation (for example, using a scraper conveyor [Iskovych-Lototsky, 2019]) in 3. The jets of water supplied through the nozzles 3 , dilute the sediment at the bottom of the sand trap and flush it to the side of the hopper 5. The sandpipers are not universal equipment for cleaning sewage from coarse-grained contaminants, they do not retain particles lighter than water. In addition, the sand trap is cumbersome and quite high energyintensive. As a rule, in modern wastewater treatment plants, they are installed between grids and primary settling tanks.

The sedimentation tanks are the simplest and cheapest, as well as the least energy-intensive type of equipment in question. However, they have significant dimensions and low productivity [Sevostianov, 2020, Vasilevskyi, 2014, Vasilevskyi, 2016]. Wastewater is supplied to the settler tank and during settling, lighter contaminants float to the surface, while heavy particles descend to the bottom of the structure, and then they are removed [Vedmitskyi, 2017].

Taking into account the above-mentioned analysis, it can be concluded that the known equipment for preliminary mechanical treatment of wastewater has significant shortcomings and requires improvement. Thus, the problem arises of developing a more efficient installation for the preliminary mechanical treatment of wastewater that meets the requirements of high productivity and reliability, low energy intensity, material intensity and cost, ensuring a high degree (95-98\%) of preliminary wastewater treatment from the coarse-grained contaminants, as well as the uninterrupted operation of the plant due to the periodic mechanized cleaning of its filter elements. This problem is solved in the proposed article. Additionally, the work gives the dependencies for determining the main operational parameters of the installation, on the basis of which a methodology for its design calculation can subsequently be developed [Sevostianov, 2019].

\section{DESCRIPTION OF THE INSTALLATION AND OPERATING PARAMETERS}

Figure 4 shows the scheme of the installation for preliminary mechanical treatment of wastewater developed by the authors. Wastewater is supplied to the unit through pipe 11 and passes through coarse gratings 14,15 to ensure the retention of large contaminants, a strip screen 4 of normal cleaning and a screen belt 3 of fine cleaning, after which the drains through the pipe are fed to the subsequent purification steps provided in accordance with the established requirements. With the continuous passage of effluents through the grilles 14,15 , the latter ones are being gradually clogged with large particles of contaminants (the maximum permissible clogging moment can be determined using the flow velocity sensors installed on the grids on the supply side). Therefore, grids 22,27 are moved to the pipe 11 to clean the grids 14, 15 (the hydraulic actuators 21, 26 provide the displacement drive of the latter). Lattices 22, 27 have the same through-holes, as in the grids 14, 15; therefore, after their extension of the

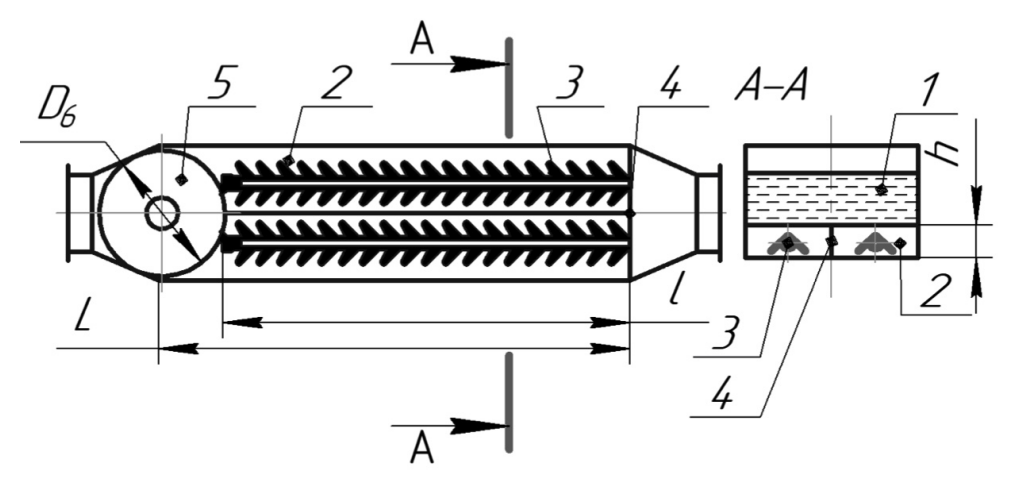

Figure 3. Schematic of a horizontal sand trap with a hydromechanical sludge removal system [Iskovych-Lototsky 2019] 


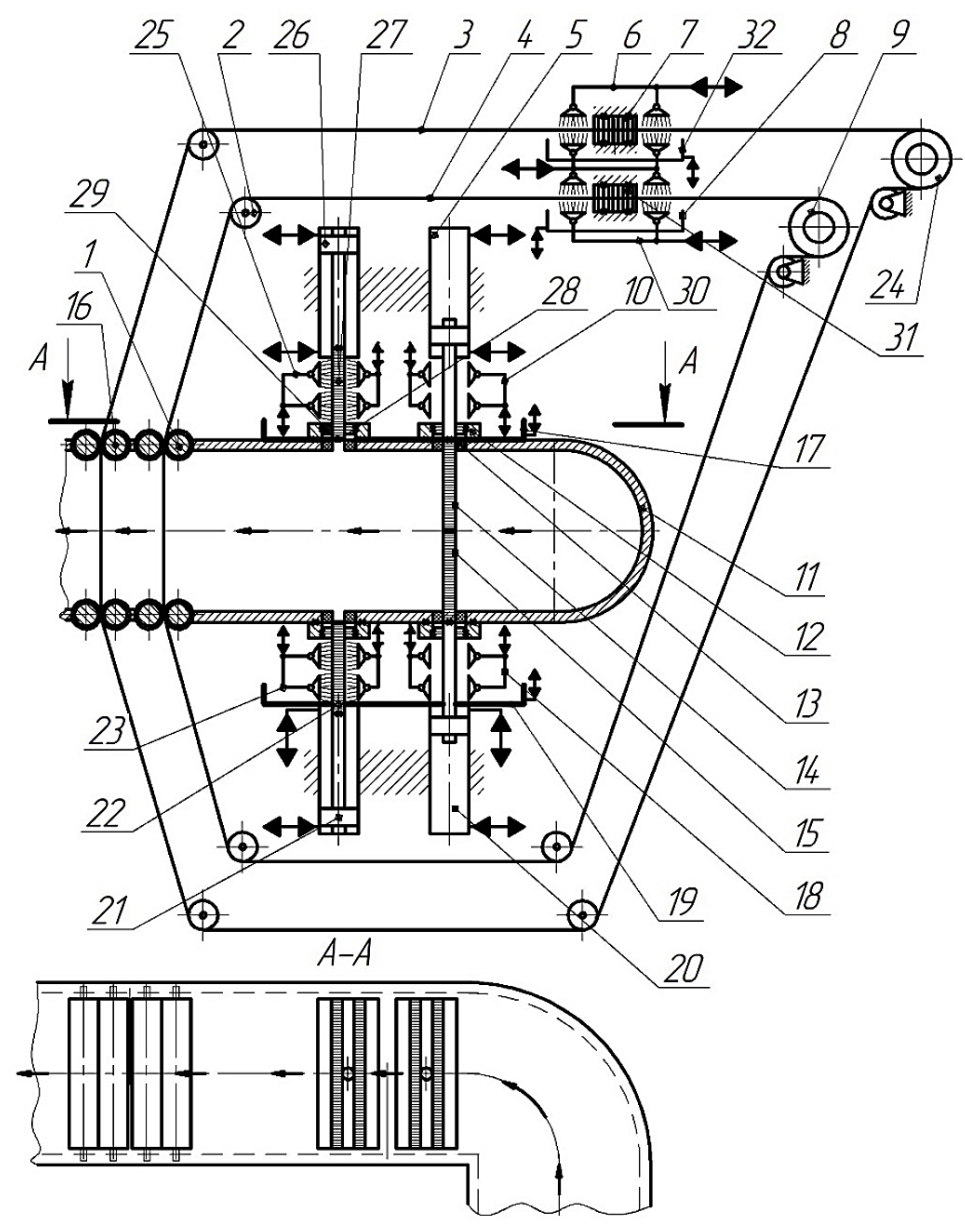

Figure 4. Scheme of installation for preliminary mechanical treatment of wastewater

grilles 14,15 , with the help of hydraulic cylinders 5,20 are withdrawn from the pipe. To this end, the pressurized working fluid is first supplied to the piston cavities of the hydraulic cylinders 21 , 26 , while their rod cavities communicate with the drain. As a result, the pistons of the hydraulic cylinders 21, 26 and, together with them, the grids 22, 27 move towards each other until the seals 28 come into contact at the ends of the grids.

Immediately after this, the working fluid begins to flow into the rod cavities of the hydraulic cylinders 5, 20 (their piston cavities are connected to the drain). Thus, the pistons and associated gratings 14,15 diverge in the opposite directions. When gratings 14, 15 are moved, they are cleaned with brushes 12 and washed with water jets from hydrants 10 . The contaminants discharged from the grids 14, 15 are drained into the sump 17, from which they are periodically withdrawn. The washed gratings 14, 15 are held by the hydraulic cylinders 5, 20 in the retracted position until the grids 22, 27 are clogged. Thereafter, the grids 14, 15 are introduced into the pipe 11 , and the grids 22, 27 are removed, cleaned with brushes 29 and washed with jets of water from the hydrants 25 . Thus, one or another pair of grids 14,15 or 22 , 26 operates in turn, ensuring continuous, highquality and high-performance pre-treatment of effluents. Smaller contaminants passing through the grilles 14,15 or 22,26 are retained by the banded sieve 4 , being connected to the ring. Depending on the intensity of its contamination, the screen is periodically or continuously moved through the pipe 11 using drum 9 with an electric drive (not shown), supporting rollers 2 and rollers 1 with a rubber working surface. Sealing of the latter in the body of the pipe 11 is carried out using rubber seals (not shown in the diagram). After the contaminated area of the screen 4 has come out of the pipe 11, as it moves, brushes 31 and hydrants 30 clean and rinse the screen. Together with water, the contaminants flow into the sump 8 . The periodic or continuous movement of the fine cleaning screen 3 provides the drive drum 24 and rubber-coated rollers 16 , and the cleaning and washing of the screen as it moves - brush 7 and hydrants 6 . The described structure of screens 3 and 4 also allows them to carry out continuous 
high-performance cleaning up to the established quality standards. The time for complete fouling off a pair of gratings 14, 15 or 22, 26 can be determined by the following formula:l

$$
t_{I}=\frac{W_{c}}{Q_{s} k_{v \cdot g} k_{c . l}}=\frac{Q_{s} k_{v . g}}{S_{p . \max } t_{i} k_{c . l}}
$$

where: $Q_{c}$ is the average flow rate of effluents passing through pipe 11;

$k_{v, g}$ - volumetric content of $1 \mathrm{~m}^{3}$ of wastewater polluted by gratings;

$W_{c}$ - the maximum permissible volume of contamination on the grids, in which it is necessary to perform their immediate cleaning;

$k_{c . l}$ - coefficient of clogging of lattices, which takes into account the area of gaps between its openings;

$S_{p . \text { rax }}$ - the maximum area of the crosssection of the grids;

$t_{i}$ - is the permissible thickness of impurities on the gratings, determined by the average cross-sectional area of the particles trapped by the gratings.

In order to prevent a significant drop in the pre-treatment performance of the effluent in the proposed installation, the gratings should be cleaned at the time $t=0.5 \cdot t_{1}$. For reception of the graphical dependence of $t_{r}, S_{p \text {.max }}$ must be taken as a part of the cross-sectional area of the pipe $-S_{p}$, which can be calculated, depending on the diameter $D_{p}$ :

$$
\begin{gathered}
S_{p \cdot \max }=0.65 \cdot S_{t}=5.1 \frac{\pi \cdot 0.1^{2}}{4} \\
S_{p \text { max }}=0.65 \frac{\pi \cdot 0.1^{2}}{4}=5.1 \cdot 10^{-3} \mathrm{~m}^{2}
\end{gathered}
$$

The flow rate $Q_{c}$ is determined based on the specified daily volume of wastewater at the enterprise $Q_{\Sigma}=500 \mathrm{~m}^{3} /$ day, the duration of shift $T_{d s}=$ $8 \mathrm{~h}$, the coefficient $k_{n p}$ that takes into consideration the duration of downtime due to the scheduled maintenance operations (cleaning, lubrication, adjustment, replacement and replenishment of working fluid in the hydraulic system, preventive testing of electrical and electronic parts of equipment, downtime for organizational and technical reasons (absence of worker, foreman, adjuster, work, raw materials, electricity, documentation, etc., as well as the coefficient variability of work $k_{v w}=3$ by the formula [Sevostianov 2019]:

$$
\begin{gathered}
Q_{c}=\frac{Q_{\Sigma}}{3600 \cdot T_{d s} k_{v w} k_{n p}}=\frac{500}{3600 \cdot 8 \cdot 3 \cdot 0.8}= \\
=7.23 \cdot 10^{-3} \mathrm{~m}^{3} / \mathrm{sec}
\end{gathered}
$$

The coefficient $k_{c . l}$ is determined based on the specified concentration of contaminants $K_{c}=$ $30000 \mathrm{mg} / \mathrm{l}$, and their density $\rho_{c}=560 \mathrm{~kg} / \mathrm{m}^{3}$ - for the meat and dairy industry. Then, for determination of the total $k_{c . I \Sigma}$ for the pollution removed at the three stages of wastewater treatment (preliminary, normal and fine treatment), the following formula can be used:

$$
k_{c . l \Sigma}=\frac{K_{c} \cdot 10^{-3}}{\rho_{c}}=\frac{30000 \cdot 10^{-3}}{560}=0.054
$$

For the meat and dairy industry in the unit volume of wastewater particles with dimensions of $10 \div 25 \mathrm{~mm}-8 \%$ by volume, dimensions of $0.5 \div 10 \mathrm{~mm}-67 \%$ and sizes $>0 \div 0.5 \mathrm{~mm}-25 \%$. Therefore, for the first stage of treatment:

$k_{c . l}=k_{c . l \Sigma} \cdot 0.08=0.054 \cdot 0.08=4.32 \cdot 10^{-3}$

It can be assumed that the maximum size of the retained particles of pollution $t_{i}=25 \mathrm{~mm}$. The coefficient $k_{3, p}$ is determined based on the diameter $D_{m}$, the size of the through cells of the plates and collets $\left(B_{n}=H_{u}=20 \mathrm{MM}\right)$. Then by the formula (1):

$$
\begin{gathered}
t_{I}=\frac{Q_{s} k_{v . g}}{S_{p . \max } t_{i} k_{c . l}}= \\
=\frac{5.1 \cdot 10^{-3} \cdot 0.025}{7.25 \cdot 10^{-3} \cdot 0.72 \cdot 4.32 \cdot 10^{-3}}=5.66 \mathrm{sec}
\end{gathered}
$$

Afterwards, $t_{I}$ was calculated under different values of $k_{c . l}=1 \cdot 10^{-3} \div 6 \cdot 10^{-3}$ (with certain changes in the composition of wastewater and the concentration of pollutants in it), as well as under changing of $Q_{s}=4 \cdot 10^{-3} \div 8 \cdot 10^{-3} \mathrm{~m}^{3} / \mathrm{sec}$ (in the cases of smaller loading of the enterprise by orders for production and accordingly smaller volumes of wastewater).

At the basis of the obtained data, graphs of $t_{I}\left(k_{v, l}\right)$ and $t_{I}\left(Q_{s}\right)$ can be obtained, as presented in Figure $5 \mathrm{a}, \mathrm{b}$. As it can be seen on the graph in Figure 5a, with increasing $k_{v} t_{I}$ actually decreases exponentially, which is due to the faster clogging of the cross-sectional areas of the gratings, with increasing concentration in wastewater of large pollutions. In such cases, it is recommended to ensure the optimal mode of operation of the installation, if possible, to reduce the flow rate $Q_{c}$ 
of wastewater to it (see Figure 5, b). If this is not possible, it is necessary to provide a similar backup installation, then both units will work with a much lower load, so the need to clean the cross-sectional areas of the gratings may occurs less frequently than in the implementation of the above-mentioned modes.

In general, any installation must have a reserve for capacity and productivity in case of these changes in the wastewater content of the enterprise.

According to Figure 5b, with increasing of $Q_{c}$, $t_{I}$ decreases exponentially and very smoothly, due to the faster passage of larger volumes of wastewater through the installation gratings and, accordingly, a larger amount of pollutions that clog faster the cross-sectional areas of the gratings. In such cases, it is also recommended to have and use an additional wastewater treatment installation at the enterprise, which will reduce the load on the first installation, ensure its operation in the optimal mode, as well as increase its reliability and resource.

The need to increase the supply of wastewater at the enterprise may arise in the case of receiving additional orders for the production of main enterprise products in a short time. This leads to additional water consumption and a corresponding increase in the volume of its effluents.

The dependence of the change in the crosssectional area of the gratings over time can be calculated by the formula:

$$
S_{p}(t)=\frac{Q_{s} k_{c . l} k_{v . g}}{t_{i}} t, 0 \leq t \leq t_{I}
$$

The time $t$ will vary from 0 to $t_{I}$ with an interval of 1 second. From the calculation results, it is possible to obtain the dependence $S_{p}(t)$ (Fig. 6). In fact, the plot shows the amount of decrease in the cross-sectional area of gratings as they are clogged until the time $t_{I}=5.66 \mathrm{sec}$, when the entire cross-section of gratings will be closed by particles of pollutions $S_{p 5}=5.1 \cdot 10^{-3} \mathrm{~m}^{2}$. The plot in Figure 6 is linear.

The change in the cross-sectional area of the gratings with time can be defined as:

$$
S_{p}(t)=\frac{Q_{s} k_{c . l}}{k_{v . g} t_{i}} t, 0 \leq t \leq t_{I}
$$

The maximum discharge of effluents through the grids can be expressed through the area of their cross-section in accordance with the following formula [Kovaleva, 2011]:

$$
Q_{s}=\mu_{l} S_{p \cdot \max } \sqrt{\frac{2 \cdot \Delta p}{\rho_{d}}}
$$

where: $\mu_{l}$ is the coefficient of flow through the lattices [Kovaleva, 2011];

$\rho_{d}$ - average density of drains;

$\Delta p$ is the pressure difference, under which the drains pass through pipe 11 (we consider it constant for a given installation). Current flow rate:

$$
Q_{s}(t)=\mu_{l}(t) \cdot S_{p}(t) \sqrt{\frac{2 \cdot \Delta p}{\rho_{d}}} ; 0 \leq t \leq t_{I}
$$

Then, on the basis of formulas $(3,4)$, we obtain:

$$
Q_{s}(t)=\frac{Q_{s}}{\mu_{l} S_{p \cdot \max }} \mu_{l}(t) \cdot S_{p}(t) ; 0 \leq t \leq t_{I}
$$

The required power of the hydraulic drive of one pair of gratings is found from the following formula [Kukharchuk, 2016]:

$$
\begin{aligned}
N_{n, p} & =\frac{2 \cdot S_{p} l_{x} p_{p}}{t_{o} \eta_{e} \eta_{p} \eta_{c} \eta_{c}}=\frac{2 \cdot S_{p} l_{x}}{t_{o} \eta_{e} \eta_{p} \eta_{c c} \eta_{c}} \frac{G_{p}-G_{p}+2 \cdot R_{y}+2 \cdot R_{b}+2 \cdot F_{m \cdot g}}{S_{p}}= \\
& =\frac{4 \cdot l_{x}\left[\mu_{f, s} \sigma_{s} S_{c s}+\mu_{f, m} \sigma_{b} S_{c, b}+2 \cdot S_{n, p} \mu_{v}\left(p_{a}+\frac{\rho_{w} v_{j}^{2}}{2}\right)\right]}{t_{o} \eta_{e} \eta_{p} \eta_{c c} \eta_{c}},
\end{aligned}
$$

where: $S_{p}$ is the effective area of the piston of the hydraulic cylinders 5, 20 from the side of the rod cavity;

$l_{x}$ - stroke length of hydraulic cylinders;

$p_{p}-$ is the nominal operating pressure at the inlet to these hydraulic cylinders;

$t_{o}$ is the duration of the opening of the gratings;

$\eta_{e^{\prime}}, \eta_{p^{\prime}}, \eta_{c c^{\prime}} \eta_{c}-$ efficiency of the electric motor of the drive, pump, coupling connecting the pump with the electric motor, the hydraulic cylinder of the drive;

$G_{p}$ is the weight of the grating 14 ;

$R_{y}^{p}$ - frictional force in the piston and rod seals of the hydraulic cylinder 5 and in the seals 13 ;

$R_{b}$ - is the frictional force when the grating 14 moves relative to the brush 12; $F_{m . g}-$ force of resistance to the movement of the grid 14, caused by the hydrodynamic head of water jets [Kovaleva, 2011] from the hydrants 10 ; 
a)

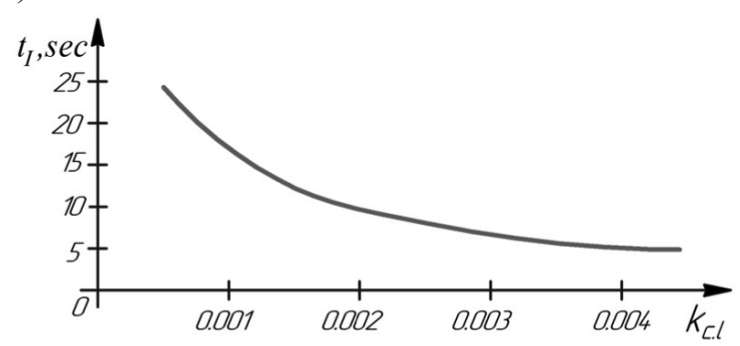

a) b)

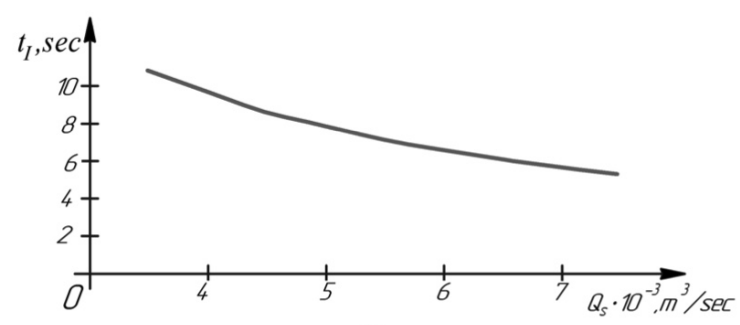

b)

Figure 5. Dependences of time $t_{I}$ of clogging of the cross-sectional areas of the installation gratings from the coefficient $k_{c . l}$ of volume content in $1 \mathrm{~m}^{3}$ of pollution detained by gratings (a) and from average expenses $Q_{s}$ of the wastewater passing through the pipe (b)

$\mu_{f, s} \mu_{f . m}-$ coefficients of friction of pairs of seal materials - metal and brushes material - metal;

$\sigma_{s^{\prime}} \sigma_{b}-$ contact stresses due to deformation of the sealant and brush;

$S_{c . S^{\prime}} S_{c . b}$ - areas of contact with seals and brushes;

$p_{a}$-atmospheric pressure;

$\rho_{w}$ - the density of water;

$V_{j}$ - average speed of jets of water from hydrants 10 ;

$S_{\text {п. }}$ - the washed area of the lattice 14;

$\mu_{v}$ - the coefficient of dynamic viscosity of water. In this case, the components $R_{y^{\prime}}$ $R_{b}, F_{m . g}$ are simple enough and can be determined experimentally with the help of a dynamometer, a prototype of the grating 14 , its seal assembly in a pipe 11 with a seal 13 , a brush 12 , hydrants 10 and a hydraulic cylinder 5 . The minimum speed of transfer of normal-cleaning tape screens 4 and fine cleaning -3 is determined from the time of their complete blockage - respectively $t_{I I}$ and $t_{I I I}$ (see also formula (1)):

$$
\begin{aligned}
& v_{\text {min. } . t}=\frac{D_{t}}{t_{I I}}=\frac{D_{t} S_{\text {s.n. } \max } t_{t . n} k_{c . n . c}}{Q_{s} k_{c . l} k_{v . s . n}} ; \\
& v_{\text {min.t }}=\frac{D_{t}}{t_{I I I}}=\frac{D_{t} S_{s . t \text { max }} t_{t . f} k_{c . f . c}}{Q_{s} k_{c . l} k_{\text {v. } . n} k_{\text {v. } . f}}
\end{aligned}
$$

where: $D_{t}$ is the diameter of tube 11 ; $k_{v . S r^{\prime}} k_{v . s . f}$ - volume content of $1 \mathrm{~m}^{3}$ of sewage sludge, retained by normal and fine cleaning sieves;

$k_{\text {c.n. } c^{\prime}} k_{c . f . c}-$ coefficients of clogging of normal and fine cleaning screens, which take into account the area of their cells;
$S_{\text {s.n.tax }} S_{\text {s.tтax }}$ - the maximum areas of a passage section of screens of normal and thin clearing for sites of the sieves which are in a pipe 11;

$t_{t . n^{\prime}} t_{t . f}$ - the average cross-sectional area of particles trapped by normal and fine cleaning sieves.

The required power on the shafts of the drums 9,24 is determined by the formulas [Kukharchuk, 2016]:

$$
\begin{aligned}
& N_{s . d . n}=\left(N_{1 b}+N_{2 b}\right) K_{a . n}+N_{d . n} ; \\
& N_{s . d . f}=\left(N_{1 s}+N_{2 s}\right) K_{a . f}+N_{d . f}
\end{aligned}
$$

where: $N_{1 b^{\prime}} N_{1 s}$ - the power spent on overcoming the sieves of the resistances created by brushes 31,7 and water jets from hydrants 30,$6 ; N_{2 b}$,

$N_{2 s}$ - the power expended for idling the sieves;

$K_{\text {a.n }} K_{a, f}$ are the coefficients that take into account the additional power costs from the resistances at the end and deflecting drums (for a conveyor length of $100 \mathrm{~m}$ and more, it can be taken to be 1 [Kukharchuk, 2017, Langthjema, 2013]);

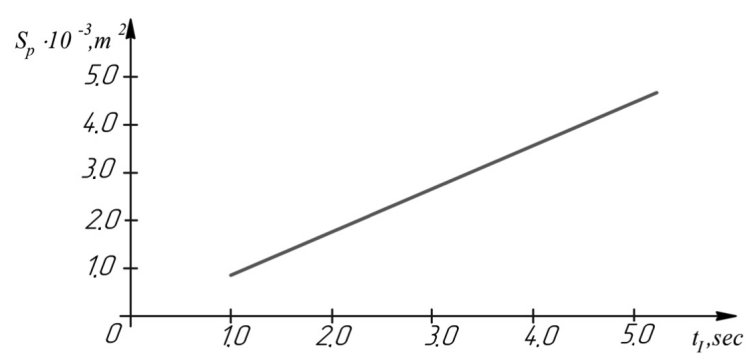

Figure 6. The dependence of the change in the crosssectional area $S_{t}$ of gratings over time 
$N_{d \cdot n^{\prime}} N_{d . f}-$ the power consumed to overcome the resistance from the presence of additional discharge devices on the sieves.

The capacities $N_{l n}, N_{l f}$ are found as (see formula (13)):

$$
\begin{aligned}
& N_{1 n}=2 \cdot v_{\text {min. } . t} \\
& {\left[\mu_{f . b . n} \sigma_{b . n} S_{c . b . n}+S_{n, p . n} \mu_{v}\left(p_{a}+\frac{\rho_{w} v_{j . n}^{2}}{2}\right)\right] ;} \\
& N_{1, f}=2 \cdot v_{\text {min. } . m}\left[\mu_{f, b . f} \sigma_{b . f} S_{c . b . f}+S_{n . p . f} \mu_{v}\left(p_{a}+\frac{\rho_{w} v_{j . f}^{2}}{2}\right)\right],
\end{aligned}
$$

where: $\mu_{f, b . r^{\prime}} \mu_{f, b . f}-$ coefficients of friction of pairs of materials of brushes 31, 7 - metal;

$\sigma_{b . n^{\prime}}, \sigma_{b . f}-$ contact stresses caused by deformations of brushes;

$S_{c . b . n^{\prime}} S_{c . b . f}$ - areas of contact with brushes;

$V_{j . n^{\prime}} V_{j . f}-$ average speeds of jets of water from hydrants 30, 6 .

The components $N_{2 n}, N_{2 f}$ are defined as [Kukharchuk, 2016]:

$$
N_{2 H}=k_{r} L_{h . n} v_{\text {min. } n} ; N_{2 f}=k_{r} L_{h . f} v_{\text {min. } f}
$$

where: $L_{h . r^{\prime}} L_{h . f}$ are the lengths of the horizontal sections of the screens 4,3 ;

$k_{r}$ is the coefficient of resistance, depending on the width of the sieves $-B_{s}$ [Kukharchuk, 2017, Langthjema, 2013], which can be found by the formula:

$$
B_{s}=D_{t}+2 \cdot t_{t}
$$

where: $t_{t}$ is the thickness of the tube 11 .

The power of the motors of the sieve drives is calculated using the dependences [Sevostyanov, 2015]:

$$
N_{m . n}=\frac{k_{i c} N_{s . d . n}}{\eta_{r}} ; N_{m . f}=\frac{k_{i c} N_{s . d . f}}{\eta_{r}}
$$

where: $k_{i c}-$ coefficient of installation capacity (power reserve);

$\eta_{\mathrm{r}}$ - efficiency of the mechanism for transfer of rotation from the electric motor to the drive drum [Tsybina, 2013, Vasilevskyi, 2014].

The above-mentioned dependences can be used to determine the usefulness of the proposed installation and its comparison with the efficiency of the known equipment for wastewater treatment.

\section{CONCLUSIONS}

The qualitative treatment of wastewater with the possibility of its safe return to the environment as well as reuse at work and at home is an urgent task for many countries. At the same time, much attention is being paid to increasing the efficiency of the cleaning process, reducing its energy consumption, as well as reducing the material intensity, complexity and equipment costs. An analysis of the known mechanical pre-treatment equipment has shown that in most cases it does not meet the modern requirements for efficiency and process continuity, as well as for water treatment quality, compactness and reliability. Taking into account these requirements, we developed a scheme of continuous mechanical three-stage wastewater treatment for which the equations and relationships have been proposed to determine the main operational characteristics of the plant: the capacity of the wastewater to be treated and the required engine power of the plant.

\section{REFERENCES}

1. Iskovych-Lototsky R.D., Ivanchuk Y.V. Veselovska N.R., Surtel W., Sundetov S. 2018. Automatic system for modeling vibro-impact unloading bulk cargo on vehicles. Proc. SPIE 10808, Photonics Applications in Astronomy, Communications, Industry, and High-Energy Physics Experiments, 1080860.

2. Iskovych-Lototsky R.D., Ivanchuk Y.V., Veselovsky Y.P., Gromaszek K., Oralbekova A. 2018. Automatic system for modeling of working processes in pressure generators of hydraulic vibrating and vibro-impact machines. Proc. SPIE 10808, Photonics Applications in Astronomy, Communications, Industry, and High-Energy Physics Experiments, 1080850.

3. Iskovich-Lototsky R., Kots I., Ivanchuk Y., Ivashko Y., Gromaszek K., Mussabekova A., Kalimoldayev M. 2019. Terms of the stability for the control valve of the hydraulic impulse drive of vibrating and vibro-impact machines. Przeglad Elektrotechniczny, 4(19), 19-23.

4. Jörg C., Mont K., Pornsak S. 2010. Response analysis of nonlinear vibro-impact system coupled with viscoelastic force under colored noise excitations. Chemical Engineering Research and Design, 88(1), 100-108.

5. Kovaleva O.M., Fomyn A.A. 2011. Methods of industrial sewage treatment. Ecology of production, $12,85-87$. 
6. Kukharchuk V.V., Hraniak V.F, Vedmitskyi Y.G., Bogachuk V.V. 2016. Noncontact method of temperature measurement based on the phenomenon of the luminophor temperature decreasing. Proc. SPIE 10031, Photonics Applications in Astronomy, Communications, Industry, and High-Energy Physics Experiments, 100312F.

7. Kukharchuk V.V., Kazyv S.S., Bykovsky S.A. 2017. Discrete wavelet transformation in spectral analysis of vibration processes at hydropower units. Przeglad Elektrotechniczny, 93(5). 65-68.

8. Langthjema M.A., Nakamurab T. 2013. Dynamics of the fluid balancer: Perturbation solution of a forced Korteweg-de Vries-Burgers equation. RIMS, Kyoto University, 1847, 73-85.

9. Sevostyanov I.V., Polishchuk O.V., Slabkiy A.V. 2015. Elaboration and research of installation for two-component vibro-blowing dehydration of food production waste. Eastern-European Journal of Enterprise Technologies. Applied mechanics, 7(77), 40-46.

10. Sevostianov I.V., Gritsun A.V., Babin I.A. 2019. Equipment for highly efficient wastewater treatment at agro-industrial enterprises. Technology, energy, transport of agro-industrial complex, 1(104), 29-39.

11. Sevostianov I.V. 2020. Technology and equipment for vibro-blowing dehydration of damp dispersive materials. Vinnytsia National Agrarian University, Vinnytsia.

12. Tsybina A.V., Diakov M.S., Vaisman Ya.Y. 2013. State and prospects of treatment and utilization of sewage sludge. Ecology and industry of Russia, 12, 56-61.

13. Vasilevskyi O.M. 2014. Methods of determining the recalibration interval measurement tools based on the concept of uncertainty. Technical Electrodynamics, 4(6), 81-88.

14. Vasilevskyi O.M. 2015. A frequency method for dynamic uncertainty evaluation of measurement during modes of dynamic operation. International Journal of Metrology and Quality Engineering, 6(2), 202.

15. Vasilevskyi O.M., Kucheruk V.Y., Bogachuk V.V. 2016. The method of translation additive and multiplicative error in the instrumental component of the measurement uncertainty. Proceedings of SPIE 10031, 1003127.

16. Vedmitskyi Y.G., KukharchukV.V., HraniakV.F. 2017. New non-system physical quantities for vibration monitoring of transient processes at hydropower facilities, integral vibratory accelerations. Przeglad Elektrotechniczny, 93(3). 69-72. 